\title{
“Digital Influencer”: Development and Coexistence with Digital Social Groups
}

\author{
Jirawat Sookkaew ${ }^{1}$ \\ Department of Computer Graphic and Multimedia \\ School of Information and Communication Technology \\ (ICT), University of Phayao (UP)
}

\author{
Pipatpong Saephoo ${ }^{2}$ \\ Department of Business Digital \\ School of Information and Communication Technology \\ (ICT), University of Phayao (UP)
}

\begin{abstract}
Digital identities, also known as virtual influencers, are created by humans through the creation of digital tools that mimic human behavior through the use of creative design. As a result of this, it has resulted in the creation of a group of people who are very fond of and trendy called "virtual influencers", particularly in the modern day. With the rise of virtual influencers, they must be used as tools in marketing and media, particularly in the online world. Because such a character is able to overcome a variety of limitations that humans are unable to provide, character styles, which do not need to have the same look or composition as people, are factors that make these characters popular, but the development of the virtual influencer depends on the social and cultural factors of the people of that era, as well as relying on technology to play a role for humans to be able to apply and use these elements to integrate with the existing virtual influencer to grow and develop more.
\end{abstract} media

Keywords_-Virtual influencer; online social; virtual character;

\section{INTRODUCTION}

Today's technology and creative innovations, such as the development of multimedia technologies, including visual and entertainment innovations, have greatly expanded human imagination and creativity. Today's technology makes it possible to create realistic images of people. The general public can create images and video materials using design software or programs from their personal computers, as well as the tools used to create them. Humans began to study and create until humans in today's society encountered and became accustomed to virtual images and 3D designs in everyday life. It appears that these computer-generated images or characters are a part of the social and media lifestyle that we see and consume daily without feeling alienated.

Human society has advanced in computer technology, which has resulted in the advancement of computer graphics, and 3D technology as byproducts of development, as well as technology in the filmmaking, animation, and game industries. Beginning the creation of 3D works, and pushing the field of 3D computer graphics to people, with the release of Star Wars in 1977, a movie that the world recognized as a visual effect work of the world. The art of creating three-dimensional works has progressed to the present day since then.

Creating an imaginary world entails much more than simply building a structure or purchasing an object. Because technology has progressed to the point where tools can generate characters or simulate people from 3D programs. Furthermore, simulated people are now easily achievable, allowing people to create and simulate characters or objects, as well as create a fictional character. "Avatar" is a term for a fictional character that is processed by software to make it more intelligent. Furthermore, this effect contributes to the creation of a more realistic display for imaginative characters. Today's technological advances have created worlds, games, and applications in which people can meet, talk, and socialize without physically being present. Individuals create virtual avatars, or representations of themselves, to represent the user in those environments [2]. Not only can the characters take on the form of people, but they can also take on the form of monsters, imaginative characters, and characters created by the creators.

\section{PROBlems Of THE STUDY}

The purpose of this research paper was to investigate the popularity of virtual influencers as well as the factors that contributed to the popularity and support of the technology trend. This includes factors that will strengthen the current trend and encourage it to progress and develop further.

\section{METHOD}

The researcher used a method to analyze data from popular virtual influencers in various forms, such as analyzing the strengths that are regarded as supporting factors in the modern era in various aspects related to lifestyle and human media use. The current technology study has the potential to help drive the technology of the digital society of virtual influencers and summarize the issues by using this method.

\section{Character DeVElopment AND Creation of FICTIONAL CHARACTERS}

Humans have long worked together in designing, thinking, and imagining. We have discovered imaginations of fictional deities dating back to recorded history, ranging from fictional paintings on cave walls to oil paintings of imaginary gods on the walls of famous artists' palaces. Visual stereotypes can appear in many different media formats: photos, movies, paintings, drawings, comics, animated movies, etc. Some of these formats allow different degrees of visual naturalism, which in the context of stereotypes is a feature of interest [1]. Visual Stereotypes and Virtual Pedagogical Agents Rather than using narrative, belief bonding, or even communication, characters are created by imagining gods or characters. The 
attentive presence of a virtual character appears to be effective in reducing subjective stress responses, especially when the user believes the virtual other is controlled by a human [9]. This is accomplished by imagining fictional characters and transforming them into images for public broadcast. For a long time, humans have been modeling the shape of creating an ideal appearance, whether it is the nature of the gods and goddesses who have a beautiful appearance according to the ideals of each nation. For a long time, stereotypes or fictional examples have existed in human society to represent the archetype of strength, beauty, and perfection that is not found in the average human being. Virtual influencers bring new scenarios to digital marketing and more; if most analyses point to more convergence than divergence between real and virtual and between human and non-human [2].

In today's world, graphic design has become more straightforward. Creating the ideal character to represent or communicate the creator's imagination or the need for a person with the desired characteristics. The use of media technologies is growing increasingly prevalent around the world; within these technologies, users are finding ways to portray themselves. The more frequently our social interaction occurs via mediation, the more common the use of avatars will become [3].

CGI (computer-generated imagery) has been a popular tool in the entertainment industry for many years. Animated graphics, figures, and characters in films and video games have changed our perceptions of our surroundings [4]. In today's world, graphic design has become more straightforward. Creating the ideal character to represent or communicate the creator's imagination or the need for a person with the desired characteristics. CGI has been a popular tool in the entertainment industry for many years. Animated graphics, figures, and characters have altered our perceptions of our surroundings, whether in film or video games [5]. Character creation, or character design, maybe another way of reflecting one's identity and the need for a human appearance can be conveyed and presented through imagination and innovation in each era.

\section{Creating a Digital Identity to Meet the REQUIREMENTS OF THE DigITAL MARKET}

The media in which humans see animated characters, monsters, and fictional characters have become the norm in today's communication and media world with the creation of human-made characters, thanks to the advancement of digital multimedia technology. There was a previous love trend when the trend of characters or cartoons flourished due to the appearance of anime characters. The following is an example of a trend that has sparked interest in the animation industry around the world, particularly in Japan, the East's leader. It is a mascot created for the Japanese voice-editing software Vocaloid, also known as Humanoid. The character's name is Hatsune Miku. A teen girl of $158 \mathrm{~cm}$ height and $42 \mathrm{~kg}$ weight appears. Her hair was turquoise, and her outfit was a short white skirt. She was adorable. This character was born in the year 2007. The Anime style, which is a technique used to create two-dimensional characters, was used to create her. The characters' popularity has spawned a massive industry phenomenon, including the ability for fans to organize their concerts utilizing 3D holographic character presentations. Furthermore, another response was their superior ability to humans' in communicating in a frequent way. This enhanced the level of intimacy with virtual influencers, showing that there were tendencies into developing bonds with them [6]. It was a way for the character to interact with her fans in person in 2014. This is yet another phenomenon in the digital world that demonstrates and conveys the fandom's affection for young girls. A large number of people also follow her on social media. More characters based on Miku's approaches and prototypes were created to satisfy the growing fan base as the character's popularity grew. This popularity has also spread outside of Japan to several other countries. This is reflected not only in the mashup of musical styles and aesthetics that underpin Hatsune's image as a performer but also in the numerous contributors to her live performance as well as her growing discography, which was created using synthesizer software [7]. Miku's success was most likely due to more than just the use of adorable new characters. Other factors that contribute to this character's popularity include Japanese culture, technology, sound, performance, release timing, appropriate publicity, and a variety of other factors. This phenomenon implies that the characters created are not always realistic, humanoid, or human representations.

The competition format and digital creations have become more realistic as we move into a more advanced era of virtual reality technology. We get to see the film characters, as well as the setting and fictional characters. In the entertainment industry, virtual reality graphics are widely used to create a more realistic appearance layout. Producers and cinematographers can express their imaginations in films by using characters created with graphics rather than human limitations. The images created by using these fictional characters are no different than those created by using real stars, people, or by creating and stimulating locations. The props are more realistic as well. It demonstrates digital characters' ability to transcend physical limitations or meet needs that humans are unable to meet. When such tools are capable of creating or producing people in such a way that it is difficult to tell them apart, creating fictional identities brings them to life. A variety of factors support and drive these factors. It is possible to make friends or form friendships in the modern era, as well as to make oneself known through a variety of social media channels. As a result, birth in the online world is regarded as a channel for presenting or informing birth. To present these digital characters, the majority of digital influencer channels currently emphasize the use of social media. Famous digital influencers were all discovered on Instagram, which is significant in and of itself. People consume content quickly on this platform, and the dimensions of the screen dictate how visual information is processed. At the start of their careers, digital influencers frequently use side channels. The online world is the primary channel for birth notification.

A persona that aligns with the target audience should be thoughtfully developed and executed. The content creators should develop an identity for the CGI that would include all components of a belief system, unique to the CGI. The CGI 
should ultimately mock a human, physically, emotionally, mentally, and spiritually. Just as the CGI and brand must align for effective advertising, as in all advertising, the brand and target audience must align. Therefore, the brand, CGI, and target audience must align [8]. Furthermore, Virtual Influencers may emerge from well-known and popular virtual characters in the digital world, such as characters from computer games, animated movies, brand characters, mascots, and so on. "It is clear that the virtual aesthetic of video games dominates this collection." When we consider heroines, or what might constitute the nature of a woman whose actions can be so courageous that she becomes superior and iconic, it becomes clear that a virtual entity integrates with Maison's founding principles. Lightning is the ideal avatar for a global, heroic woman, as well as for a world in which social networks and communications are now inextricably linked. She is also a representation of new pictorial processes. How do you create an image that deviates from traditional photography and design principles? Lightning signals the beginning of a new era of expression." Nicolas Ghesquière [9]. It not only brings popular characters from niche platforms, such as computer games, such as Final Fantasy's Lightning, but it also brings this distinct character to the world of fashion. It is also expected to continue the trend and raise awareness among other consumer groups, as well as establish a link between the brand group and the game player. This is a good way to connect relationships and create marketing channels, such as when creating the Kumamoto mascot, a black bear with red cheeks who represents Kumamoto Prefecture in Japan. Kumanmon is regarded as a pivotal figure in Kumamoto. Kumamon travels all over the world as the prefecture's sales manager, introducing people to the wonders of his hometown and its abundance of nature, seafood, and delectable fruits [10]. When people are recognized as representatives in the character, the character is a representative or image of the place, implying that the character is a representative by default. Items associated with a particular character are wellreceived and desired by both fans and customers when a fan base develops for that character. Kumamon's popularity has had its impact, with merchandise sales reaching approximately 29.3 billion Yen in the first year of its promotion into the local budget flow. This result was very close to the total income of Kumamoto Prefecture, which is well-known for its agricultural products [11].

\section{The Social DeVElopMent of Digital InFLUENCER}

The number of Virtual Influencers is growing, and the typical influencer model is to penetrate a specific market segment, such as being an influencer in one area such as travel, fashion, gaming, performances, sports, and so on. Countries or regions that wish to present language-specific content, however, the content presented retains the concept of coexistence with the characters' real life by referring to the current and imitating real life to reach or be closer to the person, for example, living like a normal person, interest in the online world like a normal person, expressing feelings or creating imitation behavior in real life based on people's age and character. It is likely that many brands will find the use of CGI as influencers appealing. The benefits are not limited to the potential of increased sales, more reach, and a reputation as an innovator. Should this be the new normal, brands may need to adjust and more CGI influencers may be needed [8]. It is thought that the character should be presented to get as close to and reach the person as possible. Although CGI allows for far more possibilities than photographic or realistic representation, these accounts attempt to depict scenes that are somewhat similar to or resemble real-life environments and scenes (Dovile Dudenaite). Human behavior may be imitated in the performance or presentation. Virtual Influencers want to sense the physical presence of the characters in the real world. After getting to know these characters, even fans and aficionados realize they are not real. The appreciation style in personality, stereotypes, or expressions, on the other hand, is most likely a big part of how people follow or become a fan base and support. CGI influencers are having an impact on the influencer marketing landscape and have the potential to change how brands communicate with their audiences [4].

\section{STRENGTHS OF VIRTUAL INFLUENCER THAT AID IN ITS GROWTH}

\section{A. Physical and Medical Limitations}

Creating a virtual influencer is certainly a character regardless of physical use or physical health, and creators can accordingly customize the character's physical characteristics, as well as the content presentation or gestures. Because computer-generated influencers are not constrained by energy levels, family commitments, or overtime legislation, they are essentially available for brand use 24 hours a day, seven days a week [12]. Clients or employers can then select one of these influencers to provide an unlimited supply of product work. These Virtual Influencers have an advantage in being a good choice for today's entertainment or advertising business due to rest or physical factors. Characters with a creative streak can take risks or create images in dangerous or difficult-to-access environments. Traditionally, brands and advertisers have used real-life models as influencers to promote their brand or product. Fashion models were chosen to be slim and beautiful. These models came with overhead costs such as hair and makeup, lodging, transportation, their own reputations and sometimes a troubled past or future. With the advent of computer-generated images (CGIs), all of those overhead costs and potential public relations crises can be eliminated [8].

\section{B. Workplace, Travel, and Time}

In today's world, where traveling or moving is difficult due to the travel health influencers' safety epidemic, visual influencers are digital information that can be displayed on modern computers and mobile phones. As a result, the budget for Virtual Influencer-related expenses has been cut. We can change the character's position to suit the task or information to be presented. The presence of software assistance and computing capabilities allows for a more realistic display, removing the need for scheduling or job queuing. We could meet Virtual Influencers on the other side of the world and then vanish in an instant. These abilities could be one of the characteristics that make influencers more appealing to their followers. 


\section{Additional Customizations to fit the Content}

Creating value or content for an influencer is simpler and less expensive than it is for the average person, as changing outfits and adding accessories around the body can change the composition in a very short time depending on the difficulty of creating a 3D piece for assembly. As a result, the new customizing influencer method saves money and time when creating content. Each image publicity for Influencers or singers, in general, necessitates a significant investment in terms of resources, expenses, and personnel. Creating content for these Virtual Influencers necessitates not only the use of computers to create images but also the character creators' skills and abilities.

\section{Bypassing Legal Constraints}

The media, labor, and the common man at work are all protected in advertising circles, as is the scope of the population's legal protection. Each region or country has its own set of specifications. They are rules that humans use to control and protect themselves to reduce problems and protect their rights. Many of these concerns are alleviated by virtual influencers: because they are not humans, there is no ethical issue of branding attached; the image can remain consistent; and the risk of indiscretions is minimized because they do not exist offline, allowing their "behavior" and image to be calibrated in the background (J. Tan). The rise of the artificial intelligence influencer: are they simply easier to work with? August 27, 2019, Marketing Interactive [13]. However, there is no specific law that governs Virtual Influencer. Despite their appearance and elements that are difficult to distinguish from the real person, the preliminary images are interpreted as a type of PR medium. The work and the virtual influencer continue to be fictitious characters, akin to cartoons or animated characters. Communication or overwork will work with these characters, so the style of content that appears with Virtual Influencers is much broader and more flexible than we've seen before. However, the requirements of different countries' media laws vary. Virtual Influencer characters are still regarded as media. Laws governing the public presentation of media also govern the rating of published media. Although CGI influencers provide brands with new ways to express themselves, the newfound power must be managed [4]. The creation of Virtual Influencer content necessitates knowledge of the countries and community's laws and customs. Even if the characters are real people, cartoons, or made-up. Although CGI influencers provide new avenues for brands to express themselves, the newfound power must be managed [12]. It is critical to have a regulatory and review body, as well as legal content related to Virtual Influencers, such as the Federal Trade Commission (FTC), whose mission is to protect consumers and competition by preventing anticompetitive, deceptive, and unfair business practices through law enforcement, advocacy, and education while not unduly burdening legitimate business activity. When receiving media, including communication from both Virtual Influencers, it is aware of information presentation and consumer protection.

\section{ACCELERATING THE FUTURE VIRTUAL INFLUENCER}

Although Virtual Influencers are gaining popularity in many parts of the world today. The character creates a distinct identity and persona to gain recognition and love from the online world as a gateway, as well as people's and brands' attention. Virtual, or rather artificial, influencers operate online in the same way that real ones do. Brands want to collaborate with them to design their fan base. Even if they are not intended to be brand ambassadors, their popularity will almost certainly attract companies looking for endorsement deals [14].

Popularity and trend in the online world are thought to be another driving factor of influencer identity, but with current trends and developments in many areas of technology, especially nowadays, it is evolving quickly. The advancement of computer graphics, as previously stated, has fueled the trend of Virtual Influencers. Online media is a factor that contributes to the trend's popularity. The Virtual Influencer's form and characteristics can be modified and developed further through the use and support of technology, which can be supplemented in the following ways.

\section{A. A.I.(Artificial Intelligence) System Development}

It is a type of technology that is vital in today's world and is used in all industries. It was created to be a simulation of the human nervous system, which processes information from learning and optimizes itself. Furthermore, it can analyze and process on its own in the process of thinking and selfdevelopment by relying on digital tools and computer systems. A field of study that seeks to explain and emulate intelligent behavior in terms of computational processes [15]. The advancement of digital human technology has a positive impact on the AI system development model. The seemingly infinite number of technologies, techniques, and applications that fall under the AI umbrella can be usefully divided into two categories. The first consists of knowledge-based systems that are "committed to the notion of generating behavior through deduction from a set of axioms" [16].

Which AI technology drives innovation in a variety of areas, including ways to accelerate advancement that can be combined with Virtual Influencer, a computer graphics applied science that can bring AI technology to promote this industry and society? In the future, the project Baby $\mathrm{X}$ will be developed as a clear example of the use of multimedia technology combined with AI processing. Dr. Mark Sagar created Baby $\mathrm{X}$, a digital artificial intelligence mixed reality installation. It is a neuro-behavioral computational model with emergent behaviors that are actively used for neuroscientific research and, at times, a public media art installation [17]. Based on the Baby X project's experiments, we can anticipate the emergence of a new type of virtual influencer in the future - a framework for interaction that could serve as a model for the next great relevant project. We can predict and see the direction of the development of existing virtual influencers or new virtual influencers from such an intriguing project, which is likely to be a tool to help foster the ability to create interactions and allow virtual influencers to meet and greet fans. Virtual Influencers, on the other hand, do not have this capability. Bringing these incredible AI systems and 
capabilities to Virtual Influencer could add augmented reality to the characters, charisma, and senses, bringing the virtual influencers to life in ways never seen before. AI is now being used by digital character creation companies to create characters for businesses, organizations, and even individuals. It was created by Soul Machines. The company is a pioneer in the field of character innovation or the simulation of real-life people.

This is a first step toward bringing Virtual multimedia and AI into the industry in the future by customers who have joined the corporate character creation when combined with the application of AI technology to be able to interact with people and study the needs of interactions to be the most similar to human personality. Vodafone ("Kiri"), NAB's UBank ("Mia"), the Ministry of Primary Industries ("Vai"), ASB Bank ("Josie"), UBS Bank ("Daniel Kalt"), and, more recently, BMW, Southern Cross, and BCG are among its customers (and digital humans) [18] and respond in an emotionally appropriate manner, providing the embodiment of the to the brand and developing brand loyalty and advocacy, bringing "humanness" into digital experiences, increasing sales conversions and customer advocacy (Monica Collier Scott Manion). Artificial Intelligence in Action: Digital Humans [18] as well as creating identity and recognition for the Brand's personality for customers and visitors. Currently, more and more such services are being born in other companies to create these digital characters, increasing the opportunity for Ai Digital Influencer to expand and widen the market, which benefits the consumer side. Consumption rises when competition in these markets rises. Nevertheless - this portrayal of the ideal can be taken one step further with interactive computer media. A key difference lies in what is otherwise seen as a central potential of virtual characters - not the least in pedagogical terms - namely their interactivity: Virtual characters may communicate, respond, and answer, thus establishing a dynamic, mutual social relation [1].

\section{B. Robotics Advancements in Industrial Technology}

We are familiar with the visualization and perception of virtual influencers' presence when viewing a digital display through a screen, but what if these influencers could transform into real people? They're just as real as robots or characters from science fiction movies. The task of perception is another important application of AI in robotics. Robots can sense their surroundings using integrated sensors or computer vision [19]. By mentioning the introduction of an AI system to manipulate and work with characters in the Virtual Character format to create a fictional character with a system that mimics human interactions as closely as possible. Virtual influencers who are unable to meet and interact with real people and followers will benefit from the adoption of this technology. Furthermore, the virtual influencer's difficulties in considering the specific context in real life were identified [6] and when considering the study and production of robots as another science that complicates. In fact, the development of these robots entails a plethora of subtleties and techniques. Humanoid robots should share the same working space as humans and should respond in a human-like manner. As a result, they require a lightweight body, high flexibility, a wide range of sensors, and a high level of intelligence [20].
Structuring is an important part of imitating and simulating a wide range of structural features, such as muscles, organs, hair, and skin, all of which necessitate the use of technology, science, engineering, art, and beauty in the creation of the humanoid. This is yet another feature that improves the Virtual Influencer industry while also introducing a new presentation style. In the future, having a unique digital identity or simply seeing these characters on screen may not be enough to meet the needs of fans, so creating a virtual influencer in the form of a robot that looks like a real person, known as a humanoid, may be required. A significant amount of difficulty is also involved, with the key being to create characters that allow for as natural an interaction and movement as possible. Robotics is a challenging field, both in terms of engineering human-like movements and expressions and the challenges that arise when a robot assumes human form. With this format, the social and emotional aspects of interaction take precedence [21]. Realbotic, a technology company focused on developing interactive and immersive virtual robots using $\mathrm{AI}$ and machine learning processes, as well as the development of external components, is currently developing augmented reality robotics in the industry. The movement and appearance of the characters create a sense of intimacy and an experience similar to meeting a real person for those who interact with them. Humanoid's creation market has grown since the company's inception. Humanoid robotics labs around the world are working to develop robots that are one step closer to the androids of science fiction. Building a humanoid robot is a difficult engineering task that necessitates a combination of mechanical, electrical, and software engineering, computer architecture, and real-time control [22]. Humanoid's growing popularity has aided the industry and related technologies in improving their capabilities in these fields. In addition to enhancing and driving the virtual influencer industry, Humanoid is involved in promoting and driving other related industries such as entertainment, film, acting, service, helping, and the puppet industry. In a variety of situations, these virtual machines can play the roles of real people.

There are also various factors that support the opportunity and development of the Virtual Influencer to increase and leap forward, such as The development of Internet sensitivity is laying the foundation for today's global systems such as technology internet 5G, Internet of thing (IoT). The presence of a new online community Social media that will support the development and innovation of multimedia technology to make it more accessible to the way of life such as the world of Metaverse. Some believe that virtual worlds will comprise a metaverse, combining immersive VR with physical actors, objects, interfaces and networks in a future form of Internet [23] a social, virtual world that parallels - and in some respects, replaces - the real world [24]. which is regarded as space and the digital world in a new platform born from the development and pushing of the future simulation technology. It also consists of many factors such as the more people have time to dive into the online world. Everyday activities are more involved in the virtual world. Creating a wide range of technological innovations increased research or the price of technology products is more accessible to each individual, etc. 


\section{Discussion}

The study's design demonstrates that the popularity of the virtual influencer's identity is driven by the trend of online technology, which provides a means to promote recognition and popularity. Today, each of the channels is becoming more widely distributed. Popular social media platforms of choice for influence are Instagram, Facebook, Snapchat, and YouTube. An influencer has the power to affect the purchase decisions of others because of their authority, knowledge, position, or relationship with their audience. It is important to note that these individuals are not simple marketing tools, but rather social relationship assets with which brands can collaborate to achieve their marketing objectives [25]. It is regarded as a factor in promoting human beings' closer and more connected relationships with virtual influencers. As the number of online media channels grows, so does the number of people who like them. The more opportunities for relationships and interactions are better.

The technology is linked to the character's development style. As processing technology and character creation have advanced, the creation of such fictional characters, such as anime, has become more feasible. However, with the advancement of digital technologies, computer generated images (CGI) are becoming more and more human-like, and virtual agents are increasingly capable of simulating human content [26]. It is thought to be another factor that has contributed to the increase in popularity and likes, as well as the broadening of its target audience, possibly including groups of fans of technology, games, cartoons, sci-fi movies, and so on. Furthermore, other factors in the creative technology industry will help promote and create success through virtual influencers by utilizing technologies throughout the world. The development of information and computer technology allows modern man to live a double or triple life, and therefore, one reality will be superimposed on another. Perhaps in the future, the virtual world and the real world will be inseparable, but these are only predictions [27].

\section{CONCLUSION}

A study of the factors and successes of virtual influencers, the phenomenon of popularity and emergence, discovered that what helps virtual influencers create characters is also due to nature's imitation and the presence of a virtual influencer. A real person can become the main character of a story or combine or improve the strengths of a variety of personalities to create a unique character. It could be a design based on the character's appearance that matches popular trends and people's preferences, or it could be designing a character that isn't necessarily based on anything that is considered personal. Virtual influencers are created by interested parties, and followers are drawn to the style and uniqueness of the content presented. Influencers are the factors that contribute to people's preferences being met. These virtual influencers are options for people who like and have an attitude toward a character's lifestyle, despite the fact that virtual influencers are not always realistic, humanistic, or display any form of perfection. That is, it could be a new social group that addresses the needs and attitudes of people who have ideas or suggestions in an era when the line between people and the digital world is almost indistinguishable. Existing influencers may be unable to replace virtual counterparts. In a technologically influenced world, it's simply a new subset of preferences and approaches. Virtual influencers are characters whose attitudes and imaginations are enriched by the attitudes or influence of a society that has adopted human-born attitudes and ideas. According to the study, the factor that contributes to popularity could be the form of popularity in appearance and the liking of the ideal character that has been with humans for a long time, such as what we see in the visualization of gods, mythological and mythological characters created to meet the needs and attitudes of demanding physical traits and desired traits. Overall support in both technology and infrastructure is an important factor that will make a difference or drive a virtual influencer's social group to gain popularity and more likes than before. The use of computers and creative technology has gained popularity for the creation of virtual influencer identities, which create beautiful characters that are similar to real people or can be conveyed as closer to humans. People's interest in information technology today coincides with a period in which both social and technological conditions are becoming increasingly involved in human daily life through active lifestyles. The media has changed, and there is still room for growth due to the rate of growth and the number of internet and electronic device users. A computing system in the field of AI that will assist virtual influencers in learning and becoming smarter. Fast Internet connection systems, IOT (Internet of Things) applications, or the robotics industry are also becoming increasingly intelligent. Furthermore, many future academic and creative innovations will help drive the combination of development with the creation of Virtual Influencer characters to grow and progress. The benefits of a virtual influencer to humans also include a variety of computer tweaks and corrections, reduced stress, and a number of physical and mental limitations that real-life celebrities face. At this point, a virtual influencer may be appropriate for activities or areas where relevant trends are likely to occur. Although a variety of factors contribute to the rise in popularity of virtual influencers, there are still many factors that virtual influencers cannot replace today. A distinct way of life in which the naturalness and presentation of content to those who follow is rather unique and communicated directly to the audience. Human development and growth continue to be so enticing that virtual influencers fail to convey these feelings to the viewers. Although virtual influencers can be customized and corrected in body proportions to be perfect or according to societal trends, the charm of change and the development of human growth is still a strength that virtual influencers cannot replace and enhance the strengths that are the hallmark of human evolution. It is an option for those who admire and sympathize with the character's way of life. That is, it could be a new social group formed to meet the needs and attitudes of people with ideas in an era when the line between people and the digital world has almost vanished. It cannot be separated, which means that the popularity of virtual influencers is derived from social behavior, attitudes, technological processes, social trends, and responses to the needs of people who are satisfied with their character and will be able to influence trends and the development of the virtual influencer 
society. In terms of living a way of life, conducting social sciences, and pushing themselves to be modern and comfortable, they are consistent and similar to humans. Because of technological advancements, life has become more convenient. Existing ones may not be able to be replaced by virtual influencers. It's simply a new subset of preferences and approaches added to the audience's options. Virtual influencers can only exist because of the encouragement and support of people who value those characteristics. Perhaps the ideal representation of the individual is where a society of virtual influencers and a variety of communication sciences collide to create new forms of self and character that satisfy and delight humans.

\section{REFERENCES}

[1] H. Magnus and G. Agneta, "Visual Stereotypes and Virtual Pedagogical Agents," Journal of Educational Technology \& Society, vol. 11, no. 4, pp. 1-15, 2008. http://www.jstor.org/stable/jeductechsoci.11.4.1.

[2] B.D.S.O. Antonio and C. Paula, "Humanized Robots: A Proposition of Categories to Understand Virtual Influencers,” Australian Journal of Information Systems, vol. 25, 2021. DOI: https://doi.org/10.3127/ajis.v 25i0.3223.

[3] J. Fox and J.A. Sun, "Avatars: portraying, exploring, and changing online and offline identities," In Handbook of research on technoself: identity in a technological society, IGI Global, 2013, pp. 255-271. DOI: 10.4018/978-1-4666-2211-1.ch014.

[4] L. S. Walter, "Changing the instagram game: the rise of a new influencer generation,” UNIVERSITY OF TWENTE , Enschede, Netherlands, 2020.

[5] K. M. Rahill and M. M. Sebrechts, "Effects of Avatar player-similarity and player-construction on gaming performance," Computers in Human Behavior Reports, vol. 4, no. 100131, p. 100131, 2021.

[6] S. N. Victoria Molin, "Robot or Human? - The Marketing Phenomenon of Virtual Influencers,” Uppsala University, Uppsala, Sweden, 2019.

[7] A. J. R. B. Cross., 10 - Keepin' it real? Life, death, and holograms on the live music stage. Chandos Publishin, 2015.

[8] C.D. Oglesby, "The New Frontier of Advertising: Computer-Generated Images as Influencers, 2019.

[9] Louisvuitton.com. [Online]. Available: https://au.louisvuitton.com/engau/articles/series-4-lightning-a-virtual-heroine-. [Accessed: 03-Oct2021].

[10] Ourkhungbangkachao.com.[Online].Available:http://www.ourkhungban gkachao.com/Uploads/articles/Tourism\%20Development\%20Case\%20S tudy\%20in\%20Kumamoto.pdf. [Accessed: 03-Nov-2021].

[11] P. Kusuma and D. W. Soewardikoen, "City mascot as A supporting force in city imaging," in Proceedings of the 4th Bandung Creative
Movement International Conference on Creative Industries 2017 (4th BCM 2017), 2018.

[12] A. V. A. Mary Caroline Creasey, "Virtual Influencing: Uncharted Frontier in the Uncanny Valley,” LUND UNIVERSITY, Lund, Sweden, 2020.

[13] "The rise of the AI influencer: Are they simply easier to work with?," Marketing-interactive.com, 27-Aug-2019. [Online]. Available: https://www.marketing-interactive.com/rise-of-ai-influencers-how-youcan-get-a-piece-of-this-pie. [Accessed: 03-Nov-2021].

[14] M. H. H. Zdenka Kadekova, "Influencer marketing as a modern phenomenon creating a new frontier of virtual opportunities," Communication Today., pp. 90-104, Jan. 2018.

[15] R. J. Schalkoff, Artificial Intelligence: An Engineering Approach. New York, NY: McGraw-Hill, 1990.

[16] N. Cristianini, "On the current paradigm in artificial intelligence," AI Commun., vol. 27, no. 1, pp. 37-43, 2014.

[17] D. Lawler-Dormer, "BABY X: Digital artificial intelligence, computational neuroscience and empathetic,” in ISEA 2013 Conference proceedings.

[18] Org.nz. [Online]. Available: https://aiforum.org.nz/wp-content/uploads/ 2019/10/FaceMe-Case-Study.pdf. [Accessed: 31-Oct-2021].

[19] Javier Andreu Perez, Fani Deligianni, Daniele Ravi and Guang-Zhong Yang, “Artificial Intelligence and Robotics,” 2016.

[20] K. Berns, T. Asfour, and R. Dillmann, "Design and control of the humanoid robot ARMAR,” in Romansy 13, Vienna: Springer Vienna, 2000, pp. 307-312.

[21] M. A. Goodrich and A. C. Schultz, "Human-robot interaction: A survey,” Found. Trends ${ }^{\circledR}$ Hum.-Comput. Interact., vol. 1, no. 3, pp. 203-275, 2007.

[22] B. Adams, C. Breazeal, R. A. Brooks, and B. Scassellati, "Humanoid robots: a new kind of tool," IEEE Intell. Syst., vol. 15, no. 4, pp. 25-31, 2000.

[23] J. Smart et al., "A cross-industry public foresight project," Metaverseroadmap.org. [Online]. Available: https://metaverseroadmap. org/MetaverseRoadmapOverview.pdf. [Accessed: 25-Oct-2021].

[24] W. Mason, "Why social VR is a huge priority for Oculus, but their metaverse is still far off," Uploadvr.com, 13-Oct-2015. [Online]. Available: https://uploadvr.com/oculus-social-vr-metaverse-palmerluckey/. [Accessed: 17-Oct-2021].

[25] S. Olenski, “The Impact Of Live Streaming On Influencer Marketing," Forbes Magazine, 25-Sep-2017.

[26] J. Arsenyan and A. Mirowska, "Almost human? A comparative case study on the social media presence of virtual influencers," Int. J. Hum.Comput. Stud., vol. 155, no. 102694, p. 102694, 2021.

[27] E. Samoylova, "Virtual world of computer games: Reality or illusion?," Procedia Soc. Behav. Sci., vol. 149, pp. 842-845, 2014. 